\title{
Asymptomatic bacteriuria among pregnant women attending antenatal care at Kilimanjaro Christian Medical Centre in Northern Tanzania
}

\author{
MOSES K. MWEI ${ }^{*}$, BARIKI MCHOME¹, BEATRICE JOHN² and EUSEBIOUS MARO ${ }^{1}$ \\ 'Department of Obstetrics and Gynaecology, Kilimanjaro Christian Medical Centre, Moshi, Tanzania \\ ${ }^{2}$ Department of Epidemiology and Biostatistics, Kilimanjaro Christian Medical University College, Moshi, \\ Tanzania
}

\begin{abstract}
Background: Asymptomatic bacteriuria has been demonstrated to have adverse maternal and pregnancy outcomes precisely pyelonephritis, low birth weight, preterm premature rupture of membranes and preterm labour.

Objective: This study aimed to determine the prevalence of asymptomatic bacteriuria and antibiotic sensitivity pattern among women attending antenatal clinic at Kilimanjaro Christian Medical Centre in Northern Tanzania.

Methodology: We conducted analytical cross sectional study involving women attending antenatal clinic at Kilimanjaro Christian Medical Centre between October and December 2016. All women who met the inclusion criteria and gave their informed consent were invited to participate. Interviews using a questionnaire were conducted to collect socio-demographic and obstetric information while urine samples were collected for laboratory processing.

Results: The prevalence of asymptomatic bacteriuria (ASB) among pregnant women in this study was 8.9\%. The organisms isolated from the urine sample according to the frequency of occurrence were Escherichia coli (50\%), Streptococcus pyogenes (19\%), Klebsiella pneumoniae (15\%), group B Streptococcus (8\%), group A Streptococcus (4\%) and Proteus mirabilis (4\%). The rate of antibiotic sensitivity among gram negative bacteria ranged from $100 \%$ among Escherichia coli and Proteus mirabilis to ceftriaxone, while Klebsiella pneumoniae and Proteus mirabilis showed no sensitivity at all to ampicillin. Among the gram positive bacteria, erythromycin was shown to have sensitivity to group A streptococcus but no sensitivity to group $\mathrm{B}$ streptococcus.

Conclusion: The prevalence of asymptomatic bacteriuria of $8.9 \%$ among pregnant women and the wide array of organisms isolated in this population warrant the development of protocols for routine ASB screening and exclusion of ampicillin as an antibiotic of choice in this cohort.
\end{abstract}

Keywords: asymptomatic, bacteriuria, pregnancy, antimicrobial sensitivity, Tanzania

\section{Introduction}

Asymptomatic bacteriuria (ASB) is defined as the presence of more than 10,000 colony forming units of the same bacteria per millilitre of urine (Ajayi et al., 2012). It is distinguished from symptomatic urinary tract infection (UTI) by the absence of symptoms compatible with a urinary tract infection (Trautner \& Grigoryan., 2014). Worldwide, surveys show that between two and ten percent of pregnant women will be diagnosed with asymptomatic bacteriuria at one point of their pregnancy (Mclsaac et al., 2005). Studies in Africa have reported very high prevalence rates such of over 40\% (Imade et al., 2010; Musbau \& Muhammad, 2013; Oladeinde et al., 2015). A relatively lower prevalence of $13 \%$ among pregnant women has been reported in a study in Mwanza, Tanzania (Masinde et al., 2009).

ASB occurs when bacteria are introduced into the urinary tract during intercourse or when wiping after bowel movement. About three quarters of ASB are caused by Escherichia coli. Other bacteria commonly associated with ASB include Klebsiella pneumoniae, Proteus species,

\footnotetext{
*Correspondence E-mail: emkyania@yahoo.com
} 
staphylococcal species, enterococci, and group B streptococci (Colgan et al., 2006; Imade et al., 2010; Jennifer et al., 2012).

The risk factors for ASB have been described to vary from one population to the other. Parity and gestational age were seen to significantly influence the prevalence of ASB in the study in Benin City, Nigeria by Oladeinde et al. (2015). While low socio economic status was positively noted as influencing the prevalence of ASB (Jalali et al., 2014). Asymptomatic bacteriuria has been associated with low birth weight, preterm labour and progression into pyelonephritis if no interventions are made (Ahmad et al., 2011). Some diseases and conditions have been associated with ASB. These included sickle cell disease, diabetes, immunosuppressibe disorders and urinary obstructions (Awolude et al., 2010; Donkor et al., 2017). It is for this reason that screening and treatment protocols have been formulated in the developed world to curb the problem.

Data available in Africa on the prevalence of ASB, associated risk factors and antibiotic sensitivity patterns are limited and widely variable and therefore not useful for use in development of similar guidelines. In Tanzania particularly, no national guidelines exist on its screening and management during pregnancy. This study aimed to determine the prevalence of asymptomatic bacteriuria and antimicrobial sensitivity pattern among women attending antenatal clinic at Kilimanjaro Christian Medical Centre in Northern Tanzania.

\section{Materials and Methods}

\section{Study site and subject}

This cross-sectional analytical study was carried out at the Kilimanjaro Christian Medical Centre located in Moshi in northern Tanzania. KCMC is a consultant and teaching hospital, offering tertiary health care with 450 bed capacity. The study involved pregnant women attending antenatal care clinic (ANC) at the Obstetrics and Gynaecology outpatient clinic between October 2016 and December 2016. The outpatient clinic runs three days a week, which is Monday, Wednesday and Friday and attends to both obstetrical and gynaecological cases with an average attendance of 300 patients per week.

Women who were attending routine antenatal visit at a gestation age less than 37 weeks criteria were recruited into the study after signing a written informed consent form. Pregnant women with a history of antibiotic use within a week prior to the interview, HIV infected clients, as well as women known to have diabetes mellitus were excluded.

\section{Data collection}

The study participants were subjected to a face to face interview to collect socio-demographic and obstetric information using a questionnaire. After the interview, a wide mouth sterile container was given to each pregnant women to collect urine sample for diagnosis of ASB. The sample was stored temporarily for an average of four hours after collection at the clinic in an ice packed cool box at temperatures of $2-8^{\circ}$ Celsius before being transferred to the laboratory. Participants who had a specific infection following processing of the results at the laboratory were treated.

Once the samples were received in the laboratory, each was examined macroscopically and recording done. Twenty microlitres of urine was placed onto blood agar and MacConkey culture media and cultured for 24 hours for gram negative and gram positive bacteria, respectively. For the samples that showed a single colony growth of 100,000 units, sensitivity to the common antimicrobial drugs used during pregnancy, was done on Muller Hinton media using a standard wire loop procedure. The drugs included ampicillin, amoxiclav, gentamicin, ceftriaxone and erythromycin. The two sets of data were then cleaned and compounded to come up with a tallying data sheet. Antimicrobial sensitivity was reported as resistant, intermediate and sensitive according to Clinical Laboratory Standard Institute (CLSI, 2015). 


\section{Data analysis}

Data were checked for completeness and accuracy prior to analysis. All data processing and analysis was performed using Statistical Package for Social Sciences (SPSS) version 20. Data on categorical variables were summarised by use of frequencies and percentages, and by means and standard deviations (SD) for continuous variables.

\section{Ethical consideration}

Ethical approval of this study was sought and granted by the Kilimanjaro Christian Medical University College Research Ethics committee (Certificate No. 969). Participants were informed about the purpose of the study and asked to sign written consent forms prior to their enrolment. Equal standard of care during attendance to the clinic and subsequent care during delivery facility was assured to the participants and non-participants of the study.

\section{Results}

\section{Socio-demographic characteristics of study participants}

A total of 304 pregnant women were involved in this study. However, data on 300 participants were analysed after 4 forms were withdrawn due to irretrievable information. The mean age of the women was 26.9 (SD 5.6) years and majority (78\%) aged 21-35 years. More than half (63\%; $\mathrm{n}=189$ ) of the women were residing in urban Moshi, $156(52 \%)$ had college or university education and majority (92\%) were married. Almost half (49\%) reported being unemployed (Table 1). Among women who had ever delivered, $168(56 \%)$ had $1-2$ deliveries, of this $73(24 \%)$ had history of abortion. More than half $(57 \% ; n=172)$ of the women were in their second trimester. Majority 151 (90\%) of the participants had never had history of delivering babies with low birth weight. About a quarter $(28 \%$ ) of the participants attended 4 or more ANC visits with $66 \%$ having had between one and three prior antenatal clinic visits. Fourteen (5\%) women had never attended ANC visit.

Table 1: Socio-demographic characteristics of participants $(\mathrm{N}=300)$

\begin{tabular}{llll}
\hline Variable & Response & Frequency & Percentage \\
\hline Age group & $15-20$ & 9 & 3 \\
& $21-35$ & 235 & 78 \\
Place of residence & 35 and above & 56 & 19 \\
& Moshi Urban & 189 & 63 \\
& Moshi Rural & 70 & 23 \\
Education level & Others & 41 & 14 \\
& Primary level & 74 & 25 \\
Occupation & Secondary level & 70 & 23 \\
& College/University & 156 & 52 \\
\multirow{3}{*}{ Marital status } & Unemployed & 146 & 49 \\
& Informal employed & 17 & 5 \\
& Formal employed & 137 & 46 \\
& Single & 25 & 8 \\
& Married & 275 & 92 \\
\hline
\end{tabular}


Table 2: Bacterial isolates $(\mathrm{N}=\mathbf{2 6})$

\begin{tabular}{lll}
\hline Bacterial Isolates & Frequency & Percentage \\
\hline Escherichia coli & 13 & 50 \\
Streptococcus pyogenes & 5 & 19 \\
Klebsiella pneumonia & 4 & 15 \\
Group B Streptococcus & 2 & 8 \\
Group A Streptococcus & 1 & 4 \\
Proteus mirabilis & 1 & 4 \\
\hline
\end{tabular}

\section{Bacterial isolates from the Urine sample}

In this study, the prevalence of asymptomatic bacteriuria among pregnant women was $8.6 \%$. The organisms isolated from the urine sample according to the frequency of occurrence were Escherichia coli (50\%), Streptococcus pyogenes (19\%), Klebsiella pneumoniae (15\%), Group B Streptococcus (8\%), Group A Streptococcus and Proteus mirabilis (4\%) (Table 2).

\section{Antimicrobial sensitivity patterns}

Antimicrobial sensitivity was highest for ceftriaxone and least for ampicillin. Escherichia coli was highly sensitive (100\%) to ceftriaxone, followed by gentamicin (75\%) and it was less sensitive to ampicillin (15\%). Klebsiella pneumoniae was not sensitive to Ampicillin however this organism had a sensitivity of $75 \%$ to ceftriaxone, gentamicin and nitrofurantoin. Proteus mirabilis was less sensitive to ampicillin as well as for nitrofurantoin. It however was $100 \%$ sensitive to ceftriaxone and gentamicin.

Table 3: Antimicrobial sensitivity rates among Gram negative bacteria

\begin{tabular}{llllll}
\hline Organism & \multicolumn{4}{l}{ Antimicrobial drug } & \\
\hline & Ceftriaxone & Nitrofurantoin & Amoxiclav & Gentamicin & Ampicillin \\
\cline { 2 - 6 } E. coli $(\mathrm{n}=13)$ & 100 & 62 & 69 & 77 & 15 \\
K. pneumoniae $(\mathrm{n}=4)$ & 75 & 75 & 75 & 100 & 0 \\
P. mirabilis $(\mathrm{n}=1)$ & 100 & 0 & 100 & 100 & 0 \\
\hline
\end{tabular}

Among the gram positive bacteria, Streptococcus group A was highly sensitive to erythromycin and ampicillin. Streptococcus group B was highly (100\%) sensitive to penicillin but less sensitive to erythromycin (Table 4). Streptococcus group A showed sensitivity to erythromycin, amoxiclav and ampicillin. Streptococcus group B displayed sensitivity to amoxiclav and ampicillin but was resistant to erythromycin. Of 5 samples that grew Streptococcus pyogenes, one colony displayed sensitivity to erythromycin while four colonies of the same organism were sensitive to amoxiclav and ampicillin. Less than half (40\%) of the Streptococcus pyogenes isolates were sensitive to penicillin. On the other hand, Streptococcus pyogenes showed a sensitivity of $20 \%$ to erythromycin.

Table 4: Antimicrobial sensitivity rates among Gram positive bacteria

\begin{tabular}{lll}
\hline Organism & & Antimicrobial drug \\
\cline { 2 - 3 } Streptococcus group $A(n=1)$ & 100 & Penicillin \\
Streptococcus group $B(n=2)$ & 0 & 100 \\
Streptococcus pyogenes $(n=5)$ & 20 & 100 \\
\hline
\end{tabular}




\section{Discussion}

Our study shows a prevalence of $8.6 \%$ of asymptomatic bacteriuria among pregnant women attending ANC at KCMC. The most common bacterial isolates were Escherichia coli followed by Streptococcus pyogenes, Klebsiella pneumoniae and Proteus mirabilis. In this study, all gram negative bacteria isolates were sensitive to ceftriaxone and gentamicin while the gram positives were sensitive to erythromycin and penicillin. The prevalence of ASB in our study is comparable to that reported in Mwanza, Tanzania (Masinde et al., 2009). Similar to other studies, this study recruited its participants from an antenatal clinic with patients with known risk factors such as recent antibiotic use and chronic urinary tract infections being excluded from the study to minimize bias (Jain et al., 2013) and therefore can explain the low prevalence. However, the prevalence was higher than that reported in a recent study in Ghana by Labi et al. (2015). On the other hand, a study in Nigeria reported much higher prevalence of ASB than that observed in our study (Oladeinde et al., 2015). The observed difference might be attributed to the characteristics of the participants in that most of the participants in this study and the Ghana study were in their third trimester of pregnancy. The clinical implication of this finding is that if screening is to be introduced in our setting, then it would be highly recommended to consider screening during the third trimester when most of the ASB positive participants were obtained.

This study was conducted at a hospital setting where trained health care providers were involved, this could have explained the lower prevalence of ASB as compared to the study done in Nigeria which was conducted at a traditional birth clinics where untrained attendants were at hand and thus contamination of samples during collection of mid-stream catch was not advocated by the untrained attendants (Oladeinde et al., 2015).

In this study, E. coli, K. pneumoniae and Group B Streptococcus were the most common organisms isolated. The finding is similar to the study done elsewhere in Tanzania (Masinde et al., 2009). The low rate of sensitivity of $E$. coli to the common first line antibiotics was low. Similar pattern of sensitivity has also been reported elsewhere in Tanzania (Masinde et al., 2009) and in West Africa (Oladeinde et al., 2015). E. coli is a normal flora of the bowel and therefore contamination of the specimen as well as contamination of the urethral area by faecal matter may contribute to its high prevalence among the asymptomatic women. The low sensitivity to the common antibiotics has been noted with concern. The implication of these findings reflects the need to enforce regulations on non-prescription use of antibiotics. This would check the growing exposure of antibiotics to these micro-organisms and thus control the rising antibiotic resistance.

There are some limitation to the findings of this study. The study was conducted in a referral centre and thus the sample may not be representative of the entire population. In conclusion, the prevalence of $8.9 \%$, coupled with the low sensitivity of microorganisms isolated in this study to first line antimicrobial agents warrant placement of national guidelines to screen and treat asymptomatic bacteriuria in this population.

\section{Disclosure}

The authors declared no conflict of interest.

\section{Author contributions}

MKM designed the study, performed the statistical analysis and participated in the writing of the manuscript. BJ provided technical assistant for statistical analysis. EM, BM and BJ contributed in reviewing the manuscript for intellectual content; MKM, EM and BM participated in project design, reviewing the manuscript and provided guidance in the statistical analysis. All authors read and approved the final manuscript. 


\section{References}

Ahmad, S., Shakooh, S., Salati, S.A. \& Muneim, A. (2011) Prevalence of asymptomatic bacteriuria among pregnant women in Kashmir. Sri Lanka Journal of Obstetrics and Gynaecology 33: 158-162.

Ajayi, A.B., Nwabuisi, C., Aboyeji, A.P., Ajayi, N.S., Fowotade, A. \& Fakeye, O.O. (2012) Asymptomatic bacteriuria in antenatal patients in Ilorin, Nigeria. Oman Medical Journal 27: 31-35.

Awolude, O.A., Adesina, O.A., Oladokun, A., Mutiu, W.B. \& Adewole, I.F. (2010) Asymptomatic bacteriuria among HIV positive pregnant women. Virulence 1 (3): 130-133.

CLSI (2015) Performance Standards for Antimicrobial Disk Susceptibility Tests. Clinical and Laboratory Standard Institute, Wayne, P.A.

Colgan, R., Nicolle, L.E., McGlone, A. \& Hooton, T.M. (2006) Asymptomatic Bacteriuria in Adults American Family Physician 74: 985-990.

Donkor, E.S., Osei, J.A., Anim-Baidoo, I. \& Darkwah, S. (2017) Risk of Asymptomatic Bacteriuria among People with Sickle Cell Disease in Accra, Ghana. Diseases 5(1): 4.

Imade, P.E., Izekor, P.E., Eghafona, N.O., Enabulele, O.I. \& Ophori, E. (2010) Asymptomatic bacteriuria among pregnant women. North American Journal of Medical Sciences 2: 263265.

Jain, V., Das, V., Agarwal, A. \& Pendey, A. (2013) Asymptomatic bacteriuria and obstetric outcome following treatment in early versus late pregnancy in North Indian women. Indian Journal of Medical Research 137: 753-758.

Jalali, M., Shamsi, M., Roozbehani, N. \& Kabir, K. (2014) Prevalence of urinary tract infection and some factors affected in pregnant women in Iran Karaj city. Middle East Journal of Scientific Research 20: 781-785.

Jennifer, P., Cyril, R., Piyumi, P., Nimesha, G., Renuka, J. (2012) Asymptomatic bacteriuria in pregnancy: prevalence, risk factors, and causative organisms. Sri Lankan Journal of Infectious Diseases 1(2): 42-46.

Labi, A.K., Yawson, A.E., Ganyaglo, G.Y. \& Newman, M.J. (2015) Prevalence and associated risk factors of asymptomatic bacteriuria in ante-natal clients in a large teaching hospital in Ghana. Ghana Medical Journal 49: 154-158.

Masinde, A., Gumodoka, B., Kilonzo, A. \& Mshana, S.E. (2009) Prevalence of urinary tract infection among pregnant women at Bugando medical centre, Mwanza, Tanzania. Tanzania Journal of Health Research 11: 154- 161.

Mclsaac, W., Carrol, J.C., Biringer, A., Bernstein, P., Lyons, E., Low, D.E. \& Permaul, J.A. (2005) Screening for asymptomatic bacteriuria in pregnancy. Journal of Obstetrics and Gynaecology of Canada 27: 20-24.

Musbau, S. \& Muhammad, Y. (2013) Prevalence of asymptomatic bacteriuria among pregnant women attending antenatal clinic at federal medical centre Nguru Yobe state. Scholars Journal of Applied Medical Sciences 1(5): 658-660.

Oladeinde, B.H., Omoregie, R. \& Oladeinde, O.B. (2015) Asymptomatic urinary tract infection among pregnant women receiving antenatal care in a traditional birth home in Benin city, Nigeria. Ethiopia Journal of Health Sciences 25: 3-8.

Trautner, B.W. \& Grigoryan, L. (2014) Approach to a positive urine culture in a patient without urinary symptoms. Infectious Disease Clinic of North America 28: 15-31. 